\title{
Protective Effect of Walnut Oil on Alcoholic Liver Disease in Mice
}

\author{
Fujun Miao', Chunlan Shan ${ }^{2}$, Wei Yang ${ }^{3}$, Hao Wang ${ }^{3}$, Shuxiang Geng ${ }^{1}$ and \\ Delu Ning ${ }^{1 *}$ \\ ${ }^{1}$ Yunnan Academy of Forestry and Grassland, Kunming, 650204, China \\ ${ }^{2}$ College of Animal Science and Technology, Yunnan Agricultural University, \\ Kunming, 650201, China \\ ${ }^{3}$ College of Veterinary Medicine, Yunnan Agricultural University, Kunming, 650201, \\ China.
}

Fujun Miao and Chunlan Shan have equally contributed.

\begin{abstract}
A B S T R A C T
Walnut oil is widely used in traditional medicine as a dietary supplement for some patients. In this study, the protective effects of walnut oil isolated from Juglans sigllata on livers of alcoholic liver disease (ALD) mice were studied. The results showed that serum alanine aminotransferase (ALT) and aspartate aminotransferase (AST) in the ethanol + walnut oil (ETH + WO) and ETH + silymarin positive group (PC) groups significantly decreased, and the lesions of ethanol-induced liver injury were relieved compared with the ethanol (ETH) group. Walnut oil significantly increased the activities of superoxide dismutase (SOD) and glutathione peroxidase (GSH-Px) in the liver of ALD. Walnut oil exerted the antiinflammatory effects in the liver of ALD by decreasing the liver index, malondialdehyde (MDA) contents, and the expression levels of F4/80. The expression levels of key factors in the toll-like receptor 4 (TLR4)/ nuclear factor- $\kappa \mathrm{B}(\mathrm{NF}-\kappa \mathrm{B})$ signaling pathway reduced significantly after feeding with walnut oil to the ALD mice. Walnut oil may exert a protective effect on ethanol-induced ALD in mice by inhibiting the TLR4/NF-אB signaling pathway.
\end{abstract}

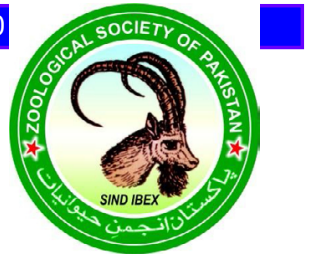

\begin{tabular}{l} 
Article Information \\
Received 23 June 2020 \\
Revised 18 October 2020 \\
Accepted 21 November 2020 \\
Available online 19 May 2021 \\
(early access) \\
Published 15 February 2022 \\
Authors' Contribution \\
FM designed the experiment and \\
drafted the manuscript. CS analyzed \\
the date and detected of AST, ALT, \\
SOD, GSH-Px and MDA contents. \\
WY prepared walnut oil and feed \\
mice. HW did liver histological \\
observation and qRT-PCR. SG did \\
immunofluorescence. DN funded \\
this study. All authors have read and \\
agreed to the published version of the \\
manuscript. \\
Key words \\
\hline Protective effect, Walnut oil, Alcoholic \\
liver disease, F4/80, TLR4/NF- $\mathrm{KB}$
\end{tabular}

\section{INTRODUCTION}

L iver is the main organ for metabolism and detoxification. Drugs, ethanol, viruses, toxins, autoimmunity, or inflammation can induce the hepatic injury, fibrosis, cirrhosis, cancer, and other diseases in the livers (Maev et al., 2014; Vega et al., 2017; Real et al., 2019). The alcoholic liver disease (ALD) is a primary consequence of heavy and prolonged drinking and contributes to the bulk of liver disease burden worldwide whose pathogenesis is still unclear and may be closely related to a variety of factors (Rocco et al., 2014). ALD is a complex process including a wide spectrum of hepatic lesions, from steatosis to cirrhosis (Louvet and Mathurin, 2015). Cell injury, oxidative stress, and inflammation are critical drivers of ethanol-induced liver injury (Arteel, 2003; Li et al., 2016). In recent years, the increasing incidence of alcoholic liver injury seriously has affected the health of humans. Therefore, the liver-protective components of safety, low toxicity, or no side effects extracted from natural products

\footnotetext{
* Corresponding author: ningdelu@163.com 0030-9923/2022/0003-1123 \$ 9.00/0

Copyright 2022 Zoological Society of Pakistan
}

have become a research hotspot (Wakimfleming and Mullen, 2005; Xu et al., 2018).

Alpha-linolenic acid (ALA) is often involved in many physiological functions such as antioxidation, anticancer, prevention of cardiovascular and cerebrovascular diseases, and brain health promotion (Simopoulos, 2002; Sijben and Calder, 2007). ALA can be converted into essential n-3 polyunsaturated fatty acids (PUFAs) in the body, increasing the levels of eicosapentaenoic acid (EPA) and docosahexaenoic acid (DHA) in the liver (Zarate et al., 2017). Walnut oil has been used widely in traditional medicine around the world, which is prescribed as a healthy high-grade food oil (Hayes et al., 2016). Walnut oil consisting of polyunsaturated fatty acids (PUFA) that includes linoleic acid (LA, C18:2n-6) and ALA (C18:3n-3), has a favorable fatty acid profile (Vinson and Cai, 2012). Walnut oil also contains functional ingredients such as phospholipids, vitamin E, melatonin, and flavonoids (Griel and Krisetherton, 2006). Walnut oil has previously been reported to reduce inflammation and oxidative stress (Willis et al., 2010; Laubertova et al., 2015).

In China, Juglans sigillata, known as Yunnan walnut, is the most cultivated walnut species. However, the hepatoprotective effect of walnut oil on acute ethanol- 
induced liver injury has not been explored extensively, whose therapeutic mechanism even remains unknown in modern medicine. In this study, walnut oil from Juglans sigllata was given to the ALD mice for detecting the index of its antioxidation and anti-inflammatory on liver injury. Furthermore, the factors in the toll-like receptor 4 (TLR4)/nuclear factor- $\kappa \mathrm{B}(\mathrm{NF}-\kappa \mathrm{B})$ inflammatory pathway involved in the development of ALD was investigated in the mice for therapeutic mechanism. The results would inform future utilization of walnut oil for the prevention of liver injury, and the formulation of functional foods.

\section{MATERIALS AND METHODS}

\section{Walnut oil preparation}

Walnut oil was obtained from Juglans sigllata cv. 'Yunxin 64' (Yunnan, China) with hydraulic cold press driven by water power. The walnut oil was filtered through a rough filter mesh and stored in brown bottles at $4{ }^{\circ} \mathrm{C}$. The contents of unsaturated fatty acid were $92.24 \%$, consisting of $20 \%$ oleic acid (OA C18: 1), 63.50\% linolenic acid (LA), and 8.74\% $\alpha$-linolenic acid (ALA).

\section{Animal experiments}

The 25 Kunming (KM) clean mice (male, 5-weekold, $22 \pm 2 \mathrm{~g}$ ) were purchased from Kunming Medical University (Certification No. SCXK (Dian) K2015-0002). All mice were provided with feed and water ad libitum. The temperature $\left(21 \pm 1{ }^{\circ} \mathrm{C}\right)$ and humidity $(45 \pm 2 \%)$ were constant in the laboratory. This study was approved by the animal protection and utilization committee of Yunnan Agricultural University. After a week of acclimation, the mice were divided into five groups (5 mice/group) randomly, including the normal control group (NC), ethanol model group (ETH), ethanol + walnut oil group $(\mathrm{ETH}+\mathrm{WO})$, walnut oil group (WO), and ETH + Silymarin (Legalon, Germany) positive group (PC). The mice in the ETH group was feed with $0.01 \mathrm{~mL} / \mathrm{g} . \mathrm{d}$ ethanol $(17.5 \%$ volumetric concentration), and the dose for the first time was double. The ETH + WO groups was fed with $2.5 \mathrm{~mL} /$ $\mathrm{kg} \cdot \mathrm{d}$ walnut oil after $30 \mathrm{~min}$ of ethanol feeding as the ETH group. The PC groups was fed with $0.2 \mathrm{~g} / \mathrm{kg} \cdot \mathrm{d}$ silymarin after $30 \mathrm{~min}$ of ethanol feeding as the ETH group. The WO group was fed with $2.5 \mathrm{~mL} / \mathrm{kg} \cdot \mathrm{d}$ walnut oil, while the NC group was fed the same dose of saline. All mice were performed intragastric administration for 14 consecutive days. Moreover, $12 \mathrm{~h}$ fasting (only drinking water) preceded the sampling process. All mice were euthanized by cervical dislocation to record gross pathological changes and collect tissue samples.

\section{Detection of serum AST and ALT contents}

After the mice were weighed, no less than $1 \mathrm{~mL}$ of blood was taken from the eyeball, and the serum was centrifuged at $3000 \mathrm{r} / \mathrm{min}$ for $10 \mathrm{~min}$. The serum was stored at $-20{ }^{\circ} \mathrm{C}$ for the next study. Alanine aminotransferase (ALT) and aspartate aminotransferase (AST) contents were detected by the dependent method according to the kit instructions (Nanjing Jiancheng Bioengineering Institute, Nanjing, China).

\section{Observation of liver histological and ultrastructure}

Part of the right lobe of the liver samples was collected from 5 mice selected randomly from each group, and embedded in paraffin. The paraffin blocks were sliced at $5 \mu \mathrm{m}$ with a sledge microtome (Typ RM 2235, Leica, Germany) and stained with hematoxylin and eosin (HE) method. Histological observations were performed at 400× magnifications (Olympus CX43 microscope, Japan).

Upon scarification of the experimental mice, part of the right lobe of the liver tissues was immediately excised on ice and fixed by immersion in $2.5 \%$ buffered glutaraldehyde for more than $4 \mathrm{~h}$. Samples were then rinsed in $0.1 \mathrm{M}$ phosphoric acid solution, post fixed in $1 \%$ osmium tetroxide, dehydrated in ethanol of ascending grades, embedded in epon, and sectioned with Ultra-thin slicer (EM-09100IS, JEOL, Japan). The ultrastructural changes were observed with the transmission electron microscope (JEM-100 CXII, JEOL, Japan) after staining with $3 \%$ uranyl acetate and lead citrate.

Detection of the liver index, activities of SOD, GSH-Px and MDA content

The weight of each mouse was recorded before dissection, and then the livers were dried with filter paper and weighed. The liver index was calculated with the recommended formula: Liver index $(\%)=$ Liver organ weight/carcass weight $\times 100 \%$.

Liver tissue $(0.5 \mathrm{~g})$ was homogenized in $4.5 \mathrm{~mL}$ saline to make $10 \%$ tissue homogenate. Then the activity of superoxide dismutase (SOD), glutathione peroxidase (GSH-Px) and malondialdehyde (MDA) in liver tissues was detected at $490 \mathrm{~nm}$ with an ELx800 ${ }^{\mathrm{TM}}$ Absorbance Microplate Reader (BioTek Instruments, Winooski, USA), according to the instructions of kits (Nanjing Jiancheng Bioengineering Institute, Nanjing, China).

\section{Immunofluorescence of liver F4/80}

Liver samples were isolated from five mice selected randomly from each group. The paraffin blocks were cut at $5 \mu \mathrm{m}$ with a sledge microtome (Typ RM 2235, Leica, Germany), and repaired with sodium citrate antigen, catalase removed with $3 \% \mathrm{H}_{2} \mathrm{O}_{2}$, blocked with normal 
goat serum. Slice was incubated overnight at $4{ }^{\circ} \mathrm{C}$ with mouse anti-F4/80 polyclonal antibody (1:800, GB11027, Servicebio Technology co., ltd, Wuhan, China). Followed by the incubation of Cy3 goat anti-rabbit $\operatorname{IgG}(1: 300$, GB21303, Servicebio Technology co., ltd, Wuhan, China) for $50 \mathrm{~min}$ at room temperature. 4', 6-diamidino-2phenylindole (DAPI) (GB1012, Servicebio Technology co., ltd, Wuhan, China) was used to stain the nucleus. The histological observations were carried out at $400 \times$ magnifications (Olympus CX43 microscope, Japan), and the images were analyzed using Image Pro-Plus 6.0 software (Media Cybernetics, Silver Spring, MD, USA).

Quantitative real time-PCR (qRT-PCR) to detect TLR4/ $\mathrm{NF}-\kappa \mathrm{B}$ genes expression

Table I illustrates the primer sequences for qRT-PCR synthesized by Beijing Tsingke ${ }^{\circledR}$ Biological Technology Corp (China). RNA was extracted from liver tissue to synthesize cDNA according to kit instructions (Tiangen Biotech Co., LTD, Beijing, China). RNA concentrations were measured with the Nanodrop 2000 spectrophotometer (NanoDrop2000c; Thermo Scientific, Waltham, MA, USA). qRT-PCR was performed with a Rotor-Gene 3000 real-time PCR cycler (Corbett/Qiagen, Hilden, Germany) and QuantiNova SYBR Green I PCR Master Mix (Qiagen, 208052, Dusseldorf, Germany). The qRT-PCR reaction condition is as follows: Predenaturation $\left(95{ }^{\circ} \mathrm{C}, 30 \mathrm{~s}\right)$, amplification $\left[\left(95^{\circ} \mathrm{C}, 5 \mathrm{~s} ; \mathrm{T}_{\mathrm{m}}, 20 \mathrm{~s} ; 72^{\circ} \mathrm{C}, 30 \mathrm{~s}\right), 40\right.$ cycles]. The final mRNA expression fold change relative to the control was normalized to $\beta$-actin.

\section{Immunohistochemistry of liver TNF- $\alpha$}

The paraffin slices of liver were prepared as described in HE method section. The immunohistochemical procedures were followed according to the instructions of Ultra-Sensitive ${ }^{\mathrm{TM}}$ SP kit-9710 and Diaminobenzidine
(DAB) color liquid manufacturers (Maixin Biotechnology products, Fuzhou, China). The slices were then incubated overnight at $4{ }^{\circ} \mathrm{C}$ with rabbit anti-TNF- $\alpha$ polyclonal antibody (1: 200, abs131997, Absin Biotechnology, Shanghai, China). The histological observations were performed at 400× magnifications (Olympus CX43 microscope, Japan), and the images analyzed using Image Pro-Plus 6.0 software (Media Cybernetics, Silver Spring, MD, USA). Expression areas of TNF- $\alpha$ of 5 views from each slice from four sections per group were counted. The average expression area of TNF- $\alpha$ per view (over areas of $0.01 \mathrm{~mm}^{2}$ ) was calculated for each group.

\section{Statistical analysis}

All data were presented as the mean $\pm \operatorname{SD}(n=3)$, and significance of differences between groups were evaluated by ANOVA (SPSS, version 23.0, SPSS Science, Chicago, IL), followed by the Duncan post-hoc test. Differences were regarded as significant and highly significant at $\mathrm{p}<$ 0.05 and $\mathrm{p}<0.01$, respectively.

\section{RESULTS}

\section{Effect of walnut oil on serum ALT and AST levels}

First, we examined whether serum ALT and AST of ALD mice were affected by walnut oil ingestion. As shown in Table II, the serum levels of ALT and AST in the ETH group increased significantly compared with the NC group ( $p<0.01)$, indicating the success of the modeling. There was no significant change in serum ALT and AST contents in the WO group ( $p>0.05)$. The serum ALT and AST levels of mice in the ETH + WO and PC groups decreased significantly compared with the ETH group (p $<0.01$ or $\mathrm{p}<0.05$ ). Indirectly, walnut oil could reduce the liver injury of ALD mice.

Table I. Specific primers for target genes.

\begin{tabular}{|c|c|c|c|}
\hline Name & Genbank No. & Sequences (5' -3') & $\mathrm{T}_{\mathrm{m}}\left({ }^{\circ} \mathrm{C}\right)$ \\
\hline \multirow[t]{2}{*}{$\beta$-actin } & XM_005887322.2 & F-CCTGCGGCATTCACGAAACTAC & 59.5 \\
\hline & & R-ACTCCTGCTTGCTGATCCACAATC & \\
\hline \multirow[t]{2}{*}{ TLR4 } & XM_032902869.1 & F-AGGCAGCAGCTCGAATTGTATC & 55.0 \\
\hline & & R-TTCCATCCAACAGGGCTTT & \\
\hline \multirow[t]{2}{*}{$N F-\kappa B$} & AF079314.2 & F-GACGATCTGTTTCCCCTCAT & 57.4 \\
\hline & & R-GCTTCTCTCCCCAGGAATAC & \\
\hline \multirow[t]{2}{*}{$T N F-\alpha$} & AB185894.1 & F-GGCAGGTTCTGTCCCTTTCA & 58.0 \\
\hline & & R-CTGTGCTCATGGTGTCTTTTCTG & \\
\hline \multirow[t]{2}{*}{$I L-1 \beta$} & NM_008361.4 & F-AATGAAAGACGGCACACCCA & 57.4 \\
\hline & & R-GGAAGACAGGCTTGTGCTCT & \\
\hline
\end{tabular}


Table II. Effect of walnut oil on serum alanine aminotransferase (ALT) and aspartate aminotransferase (AST) levels of ALD mice.

\begin{tabular}{lll}
\hline Groups & ALT (U/L) & AST (U/L) \\
\hline $\mathrm{NC}$ & $23.88 \pm 1.89 \mathrm{Aa}$ & $44.96 \pm 1.94 \mathrm{Aa}$ \\
$\mathrm{ETH}$ & $38.56 \pm 3.17 \mathrm{Cc}$ & $65.70 \pm 2.52 \mathrm{Cc}$ \\
$\mathrm{ETH}+\mathrm{WO}$ & $33.23 \pm 2.39 \mathrm{BCb}$ & $58.43 \pm 2.43 \mathrm{Bb}$ \\
$\mathrm{WO}$ & $22.91 \pm 1.63 \mathrm{Aa}$ & $43.20 \pm 2.07 \mathrm{Aa}$ \\
$\mathrm{PC}$ & $30.07 \pm 2.10 \mathrm{ABb}$ & $55.41 \pm 2.07 \mathrm{Bb}$ \\
\hline
\end{tabular}

Note: Data are presented as averaged expression levels and standard deviation of three repeats. Different uppercase letters (A, B, C, D) and lowercase letters $(\mathrm{a}, \mathrm{b}, \mathrm{c}, \mathrm{d})$ compared between different groups indicate the significant difference with $p<0.01$ and $p<0.05$, respectively. The same letters compared between different groups indicate no significant difference $(p>0.05)$. The same below.

$\mathrm{NC}$, nominal control; ETH, ethanol group; WO, walnut oil group; PC, positive control.

Effect of walnut oil on histological and ultrastructural changes in liver

Considering the apparent effect of walnut oil on serum ALT and AST, we further investigated if the histological changes of liver are different among the four groups. As shown in Figure 1, HE staining results can be observed that the structure of the hepatic lobules and endothelial cells in the central vein were clear, the hepatic cells arranged neatly around the central vein in a radial manner, the hepatic cords arranged neatly, and the hepatic sinuses were seen in the NC and WO group. There was no degeneration and necrosis or inflammatory cell infiltration in the cells, and no histopathological changes were observed in the NC and WO group. Compared with the NC group, the ETH group showed liver tissue structure disorder, central vein enlargement, hepatocyte edema, steatosis, necrosis, and other phenomena in the hepatocytes, presenting cavitation of different sizes, inflammatory cell infiltration in the portal area, and some false lobule. Compared with the ETH group, the ETH+WO and PC groups showed a slight improvement in hepatocytes and hepatic cord lesions, with less fatty degeneration and less inflammatory cell infiltration.

As shown in Figure 1, the normal morphology of mice liver cells can be observed in the NC group with TEM. The cell nucleus assumes the circular with the euchromatin distributed evenly, and the cell membrane was intact and smooth. Mitochondria had a clear double membrane and crest structure. The rough endoplasmic reticulum (RER) was neat and enriched visible ribosome particles. There was no ribosomes and lipid attached on the smooth endoplasmic reticulum (SER). However, the nuclei of the liver cells in the ETH group were irregular. For example, the nuclear membrane was uneven, the nuclear pores were increased significantly, the perinuclear gap appeared, the mitochondria malformed, the ridge arrangement was disordered, the rough endoplasmic reticulum was swollen, the ribosomal particles fell off, and a large number of lipid droplets could be seen. In the $\mathrm{ETH}+\mathrm{WO}$ and PC groups, compared with the ETH group, the symptoms of the ultrastructural changes in mouse liver cells were improved. The nuclear membrane was intact, of which only a small part was irregularly serrate. The mitochondrial was restored to contain clear crest and double-layer membrane structure with slight swelling. There were slight endoplasmic reticulum swelling and ribosome shedding, and a small number of lipid droplets were observed. The liver cells of the WO group were consistent with those of the blank control group, with normal structure and no ultrastructural changes.

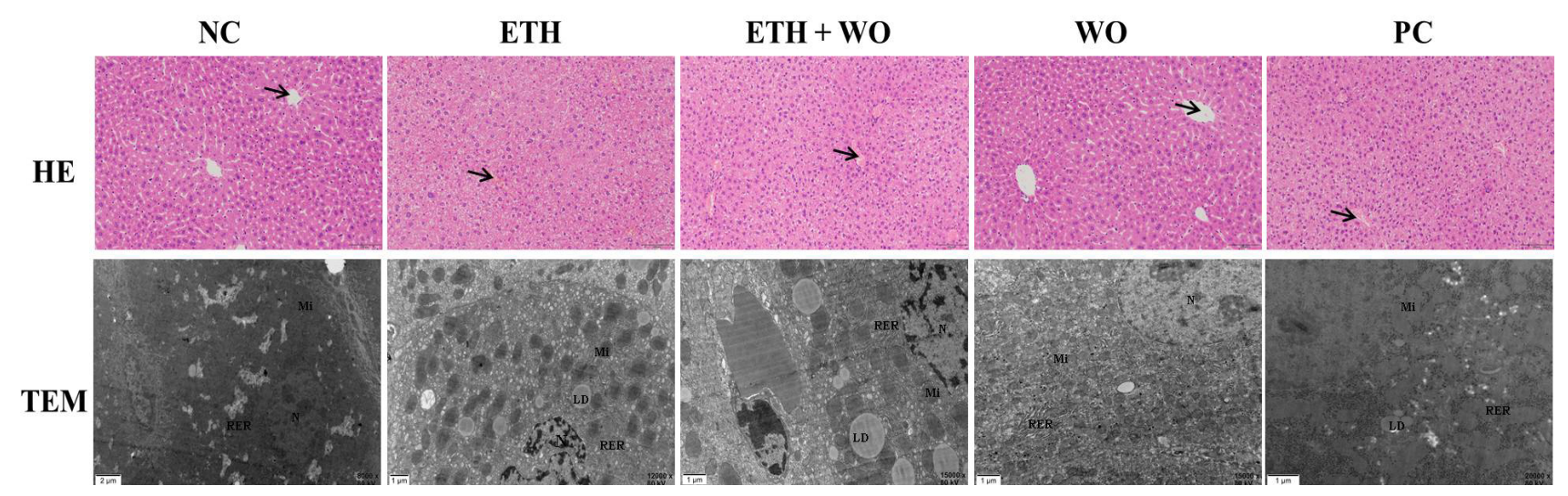

Fig. 1. Effect of walnut oil on liver morphology (200× magnification; HE staining) and ultrastructure (TEM)of ALD mice. N, nucleus; RER, rough endoplasmic reticulum; Mi, mitochondria; LD, lipid droplets.

For details of groups, see Table II. 
Table III. Effect of walnut oil on the liver index, activities of SOD, GSH-Px and MDA content.

\begin{tabular}{lllll}
\hline Group & Liver index & SOD $(\mathbf{U} / \mathbf{m g})$ & GSH $(\mathbf{U} / \mathbf{m g})$ & MDA (n mmol/mg) \\
\hline $\mathrm{NC}$ & $4.21 \pm 0.14 \mathrm{Aa}$ & $219.05 \pm 11.44 \mathrm{Bc}$ & $545.15 \pm 17.38 \mathrm{CDc}$ & $4.22 \pm 0.12 \mathrm{Aa}$ \\
$\mathrm{ETH}$ & $4.74 \pm 0.13 \mathrm{Bc}$ & $159.64 \pm 9.11 \mathrm{Aa}$ & $433.36 \pm 14.53 \mathrm{Aa}$ & $5.29 \pm 0.11 \mathrm{Cc}$ \\
$\mathrm{ETH}+\mathrm{WO}$ & $4.58 \pm 0.10 \mathrm{ABbc}$ & $190.10 \pm 10.66 \mathrm{ABb}$ & $477.16 \pm 12.14 \mathrm{ABb}$ & $4.82 \pm 0.16 \mathrm{BCb}$ \\
$\mathrm{WO}$ & $4.23 \pm 0.10 \mathrm{Aa}$ & $223.01 \pm 16.38 \mathrm{Bc}$ & $564.15 \pm 18.18 \mathrm{Dc}$ & $4.10 \pm 0.15 \mathrm{Aa}$ \\
PC & $4.34 \pm 0.12 \mathrm{ABab}$ & $195.28 \pm 9.13 \mathrm{ABbc}$ & $500.80 \pm 14.95 \mathrm{BCb}$ & $4.61 \pm 0.26 \mathrm{ABb}$ \\
\hline
\end{tabular}

For details of groups, see Table II.

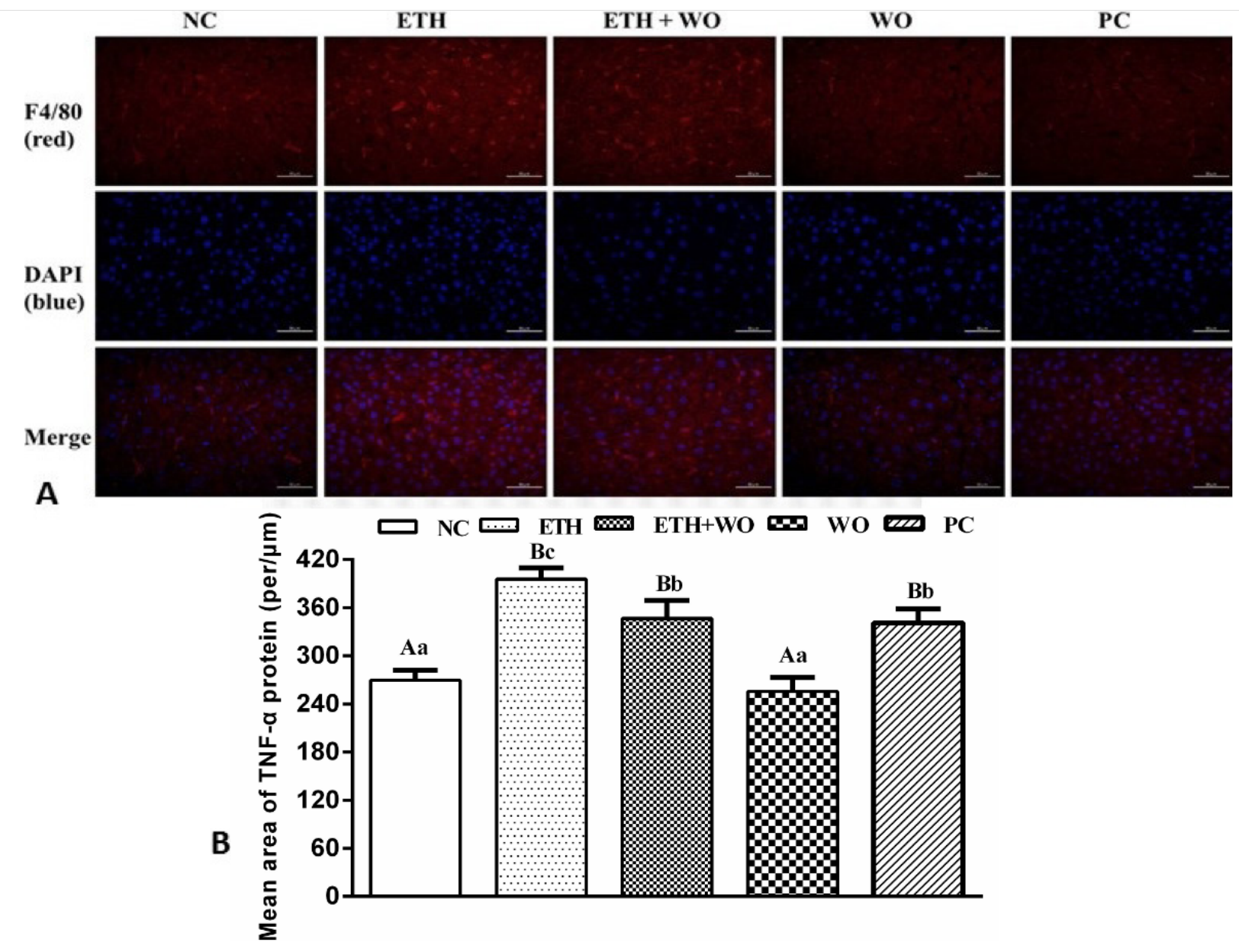

Fig. 2. Effects of walnut oil on the expression of F4/80 in liver tissue of mice. A, Identification of F4/80 expression in liver by immunofluorescence (400× magnification; F4/80 staining, red and nuclear staining, blue); B, The F4/80 positive cells rate in liver. Note: Data are presented as averaged expression levels and standard deviation of three repeats. Different uppercase letters (A, B, $\mathrm{C}, \mathrm{D})$ and lowercase letters ( $\mathrm{a}, \mathrm{b}, \mathrm{c}, \mathrm{d})$ compared between different groups indicate the significant difference with $\mathrm{p}<0.01$ and $\mathrm{p}$ $<0.05$, respectively. The same letters compared between different groups indicate no significant difference $(p>0.05)$. The same below.

For details of groups, see Table II.

Effect of walnut oil on the liver index, activities of SOD, GSH-Px, and MDA content of liver

The levels of SOD, GSH-Px, and MDA are indicators of oxidative stress. As illustrated in Table III, compared with the NC group, the liver index in the ETH group increased significantly $(\mathrm{p}<0.01)$, the activity of SOD and GSH-Px decreased significantly $(\mathrm{p}<0.01)$, and the content 
of MDA increased significantly $(\mathrm{p}<0.01)$. Compared with the ETH group, the liver index in the ETH + WO group decreased insignificant $(p>0.05)$. The activity of SOD and GSH-Px increased $(p<0.05)$, while the content of MDA decreased significantly compared with the ETH group ( $p$ $<0.01)$. The liver index, SOD, GSH-Px, and MDA in the WO group were not significantly different from those in the $\mathrm{NC}$ group $(\mathrm{p}>0.05)$. It was suggested that walnut oil could significantly improve the liver organ index, the activity of SOD, and GSH-Px as well as reduce the content of MDA. Besides, it could enhance the antioxidant capacity, improve the level of lipid peroxidation in the liver of ALD mice effectively, and reduce the damage of alcohol.

\section{Effect of walnut oil on the liver F4/80}

As seen in Figure 2A, F4/80 was the surface marker of kupffer cells, and the positive cells were expressed in red. There was little F4/80 positive expression in the NC group, while F4/80 positive expression increased significantly was found in the ETH group ( $p<0.01$ ) (Fig. 2B). The expression of $\mathrm{F} 4 / 80$ in the WO group was not statistically significant compared with that in the NC group $(\mathrm{p}>0.05)$. Compared with the ETH group, the expression of F4/80 in the ETH+WO and PC groups reduced significantly $(\mathrm{p}<$ 0.01 ). The results showed that walnut oil could reduce the expression level of F4/80 in damaged liver tissue.

Effect of walnut oil on the relative genes expression of liver TLR4/NF- $\kappa B$ signal pathway in mice

We further attempted to determine the effect of walnut oil on the TLR4/NF- $\kappa$ B signaling pathway in ALD mice. TLR $4, N F-\kappa B$, TNF- $\alpha$, and IL- $1 \beta$ were chosen as the TLR4/NF- $\kappa \mathrm{B}$ signal pathway key factors, whose transcription levels were increased significantly in the ETH group compared with the NC group ( $p<0.01)$ (Fig. $3)$. Compared with the ETH group, the expression levels of TLR4 and NF- $\kappa \mathrm{B}$ gene in the liver of the ETH+WO and $\mathrm{PC}$ groups reduced significantly $(\mathrm{p}<0.01)$, and that of TNF- $\alpha$ and IL- $1 \beta$ mRNA reduced significantly $(p<0.05)$. Transcription levels of these key genes in the TLR4/NF$\kappa \mathrm{B}$ signal pathway did not alter significantly in the WO group $(\mathrm{p}>0.05)$.

\section{Effect of walnut oil on the liver TNF- $\alpha$ protein expression}

We further examined the expression of proinflammatory cytokine TNF- $\alpha$ in ALD mice (Fig. 4A). As seen in Figure 4B, the mean areas of the ETH group were increased significantly compared with the NC group $(\mathrm{p}<0.01)$. Compared with the ETH group, the mean areas of TNF- $\alpha$ in the ETH+WO and PC groups reduced significantly $(p<0.05)$. The expression of TNF- $\alpha$ in the WO group was not statistically significant compared with that in the $\mathrm{NC}$ group $(\mathrm{p}>0.05)$. The results suggest that walnut oil may reduce protein expression of TNF- $\alpha$, hence improve the anti-inflammatory function.

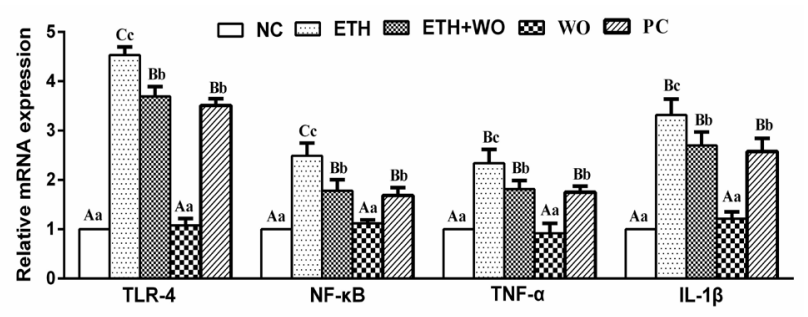

Fig. 3. The relative expression of TLR4, NF- $\kappa B, T N F-\alpha$, and IL- $1 \beta$ mRNA in the liver. For details of groups, see Table II.

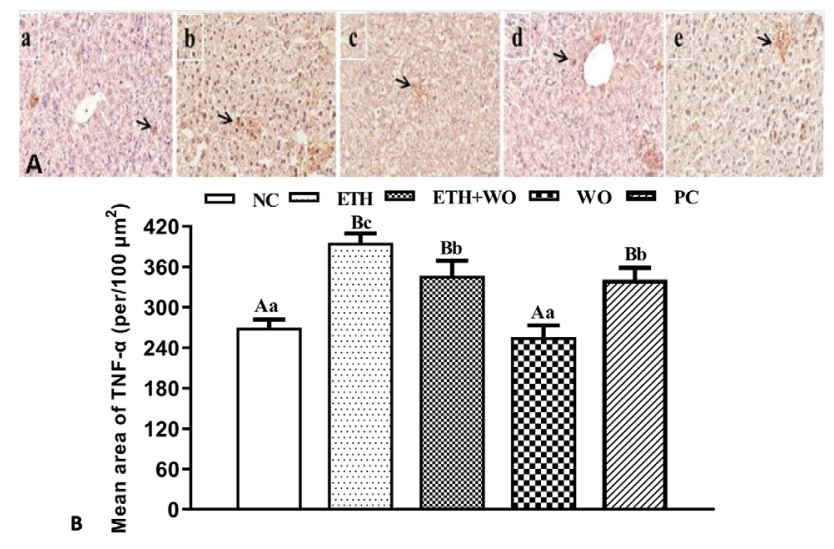

Fig. 4. Effects of walnut oil on the expression of TNF- $\alpha$ in liver tissue of mice. A, TNF- $\alpha$ protein expression in liver by immunohistochemistry ( $400 \times$ magnification) (a, $\mathrm{NC}$; b, ETH; c, ETH + WO; d, WO; e, PC); B, The IOD value of TNF- $\alpha$ protein in liver.

For details of groups, see Table II.

\section{DISCUSSION}

Walnut oil has antioxidant, anti-inflammatory, antitumor, and other pharmacological effects (Hayes et al., 2016). To the best of our knowledge, this was the first study to investigate the beneficial properties of walnut oil from Juglans sigllata on ethanol-induced liver disease. The liver is the main organ of alcohol metabolism in the body. Alcohol and its metabolites (acetaldehyde) will damage the liver tissue structure, cause metabolic abnormalities, aggravate the oxidative stress, and accelerate the release of inflammatory factors (Chao et al., 1997). Alanine aminotransferase (ALT) aspartate aminotransferase (AST) are commonly used in the clinical evaluation of liver function (Wang et al., 2015). ALT and AST are 
non-specific intracellular functional enzymes that exist in liver cells and are released into the blood when liver cells are damaged (Reichling and Kaplan, 1988). Silymarin protects against the liver injury caused by acute ETH administration. In view of its nontoxic nature, it may be developed as an effective therapeutic agent for alcoholinduced liver disease by its antioxidative stress and antiinflammatory features (Song et al., 2010). Silymarin was used as a positive control in this study. In our findings, walnut oil reduced the activity of serum ALT and AST and liver index of ALD mice significantly as well as the degree of pathological damage of liver tissue structure, indicating that liver function was improved to some extent.

Catabolism of alcohol in the liver will promote the production of reactive oxygen species (ROS). Excessive intake of alcohol will aggravate the oxidative stress of the liver, resulting in the excessive accumulation of ROS in the body the lipid peroxidation of the liver cell membrane and the damage to the liver cells. SOD and GSH-Px are necessary antioxidant enzymes in ROS scavenging, whose activity in the antioxidant enzyme system of the organism reflects the ability of the organism to scavenge free radicals indirectly (Selvaratnam and Robaire, 2016). SOD is an essential enzymatic antioxidant in the body, which can promote the transformation of superoxide free radicals into hydrogen peroxide and prevent lipid peroxidation in biofilms. GSH-Px can also remove superoxide free radicals in the body and protect cells from oxidative damage (Deng et al., 2012).

However, the toxic acetaldehyde produced by ethanol metabolism can reduce the level of SOD and GST-px, enhance the toxicity of free radicals, and then cause lipid peroxidation and promote cell apoptosis (Chen et al., 1999). As the final product of lipid peroxidation, MDA is one of the indicators reflecting the over-strong oxidation in tissue damage. The more MDA existing in tissue, the higher the oxidative activity, and the more severe tissue damage will occur (Sid et al., 2013). In this study, the activities of SOD and GSH-Px in the alcoholic liver of mice increased significantly, and the content of MDA decreased after administering walnut oil. The results implied that walnut oil had a significant antioxidant effect, which improved the ability to eliminating excessive free radicals of the liver. Our findings were consistent with the results of Zhao et al. that walnut oil increases the enzyme activity of SOD and GSH-Px significantly, and has antioxidant properties of reducing the oxidative stress (Zhao et al., 2018).

Long-term excessive drinking can raise the intestinal permeability and the LPS serum levels as well as produce the endotoxin to specific receptors on the surface of liver Kupffer cells. The active TLR4/NF- $\mathrm{B}$ inflammatory pathway releases the inflammatory factors, such as NF- $\kappa \mathrm{B}, \mathrm{TNF}-\alpha$, and IL- $1 \beta$, which induced liver cell necrosis and apoptosis and made liver tissue inflammation and fibrosis (Li et al., 2013). Therefore, the expression levels of inflammatory factors in the liver tissue can reflect the degree of hepatocyte injury and inflammatory response (Basak and Hoffmann, 2008). Kupffer cells in the liver are essential defense cells involved in liver repair by releasing various inflammatory mediators (Bala et al., 2017). F4/80 is one of the marker proteins of kupffer cells, whose expression level could reflect the degree of hepatocyte injury and inflammatory response (Elchaninov et al., 2019). In this study, walnut oil was found to reduce the expression level of F4/80 protein in the liver of ALD mice significantly. Alcohol activates the TLR4/NF- $\kappa$ B signaling pathway in liver macrophages, releasing large amounts of inflammatory cytokines (Hritz et al., 2008). This study further examined the effects of walnut oil on the TLR4/ $\mathrm{NF}-\kappa \mathrm{B}$ signaling pathway in ethanol-induced liver injury and found that walnut oil inhibited the expression of key factors in the TLR4/NF- $\mathrm{BB}$ signaling pathway. Long-term consumption of walnut oil may antagonize inflammatory damage.

\section{CONCLUSION}

In conclusion, walnut oil could reduce the serum ALT and AST levels, increase the activities of antioxidant enzymes in the liver, reduce liver F4/80 protein, and inhibit the expression levels of key genes in the TLR4/NF$\kappa \mathrm{B}$ signaling pathway in ALD mice. It is suggested that walnut oil had an excellent safety-benefit ratio in ALD. People who drink alcohol for a long time can protect the liver with walnut oil.

\section{ACKNOWLEDGEMENTS}

This work was financially supported by the Science and Technology Major Program of Yunnan Province, China (Grant No.2018ZG003).

\section{Ethics statements}

All animals were kept in a pathogen-free environment and fed ad lib. This study was approved by the Institutional Animal Care and Use Committee of Yunnan Agricultural University, and all applicable institutional and governmental regulations concerning the ethical use of animals were followed.

\section{Statement of conflict of interest}

The authors have declared no conflict of interest. 


\section{REFERENCES}

Arteel, G.E., 2003. Oxidants and antioxidants in alcoholinduced liver disease. Gastroenterology, 124: 778790. https://doi.org/10.1053/gast.2003.50087

Bala, S., Csak, T., Kodys, K., Catalano, D., Ambade, A., Furi, I., Lowe, P., Cho, Y., Irachetavellve, A. and Szabo, G., 2017. Alcohol-induced mir-155 and hdac11 inhibit negative regulators of the tlr4 pathway and lead to increased lps responsiveness of kupffer cells in alcoholic liver disease. J. Leukoc. Biol., 102: 487-498. https://doi.org/10.1189/ jlb.3A0716-310R

Basak, S. and Hoffmann, A., 2008. Crosstalk via the nf-kb signaling system. Cytokine Growth Factor Rev., 19: 187-197. https://doi.org/10.1016/j. cytogfr.2008.04.005

Chao, Y., Young, T., Tang, H. and Hsu, C., 1997. Alcoholism and alcoholic organ damage and genetic polymorphisms of alcohol metabolizing enzymes in chinese patients. Hepatology, 25: 112117. https://doi.org/10.1002/hep.510250121

Chen, J., Robinson, N.C., Schenker, S., Frosto, T.A. and Henderson, G.I., 1999. Formation of 4-hydroxynonenal adducts with cytochrome c oxidase in rats following short-term ethanol intake. Hepatology, 29: 1792-1798. https://doi. org/10.1002/hep.510290611

Deng, X., Wang, S., Deng, A., Liu, B., Edgerton, S.M., Lind, S.E., Wahdanalaswad, R. and Thor, A.D., 2012. Metformin targets stat3 to inhibit cell growth and induce apoptosis in triple-negative breast cancers. Cell Cycle, 11: 367-376. https://doi. org/10.4161/cc.11.2.18813

Elchaninov, A.V., Fatkhudinov, T., Vishnyakova, P.A., Lokhonina, A.V. and Sukhikh, G.T., 2019. Phenotypical and functional polymorphism of liver resident macrophages. Cells, 8: 1032. https://doi. org/10.3390/cells8091032

Griel, A.E. and Krisetherton, P.M., 2006. Tree nuts and the lipid profile: A review of clinical studies. $B r$. J. Nutr., 96(S2):S68-S78. https://doi.org/10.1017/ BJN20061866

Hayes, D., Angove, M.J., Tucci, J. and Dennis, C., 2016. Walnuts (Juglans regia) chemical composition and research in human health. Crit. Rev. Fd. Sci. Nutr., 56: 1231-1241. https://doi.org/10.1080/10408398. 2012.760516

Hritz, I., Mandrekar, P., Velayudham, A., Catalano, D., Dolganiuc, A., Kodys, K., Kurtjones, E.A. and Szabo, G., 2008. The critical role of toll-like receptor (tlr) 4 in alcoholic liver disease is independent of the common tlr adapter myd88. Hepatology, 48: 1224-1231. https://doi.org/10.1002/hep.22470

Laubertova, L., Koňarikova, K., Gbelcova, H., Durackova, Z. and Žitňanova, I., 2015. Effect of walnut oil on hyperglycemia-induced oxidative stress and pro-inflammatory cytokines production. Eur. J. Nutr., 54: 291-299. https://doi.org/10.1007/ s00394-014-0710-3

Li, R., Xu, L., Liang, T., Li, Y., Zhang, S. and Duan, $\mathrm{X}$., 2013. Puerarin mediates hepatoprotection against ccl4-induced hepatic fibrosis rats through attenuation of inflammation response and amelioration of metabolic function. Fd. Chem. Toxicol., 52: 69-75. https://doi.org/10.1016/j. fct.2012.10.059

Li, S., Hong, M., Tan, H., Wang, N. and Feng, Y., 2016. Insights into the role and interdependence of oxidative stress and inflammation in liver diseases. Oxid. Med. Cell. Longev., 2016: 4234061-4234061. https://doi.org/10.1155/2016/4234061

Louvet, A. and Mathurin, P., 2015. Alcoholic liver disease: Mechanisms of injury and targeted treatment. Nat. Rev. Gastroenterol. Hepatol., 12: 231-242. https://doi.org/10.1038/nrgastro.2015.35

Maev, Abdurakhmanov, Andreev and Dicheva, 2014. Alcoholic liver disease: State of the art. Ter. Arkh., 86: $108-116$.

Real, M., Barnhill, M.S., Higley, C., Rosenberg, J. and Lewis, J.H., 2019. Drug-induced liver injury: Highlights of the recent literature. Drug Saf., 42: 365-387. https://doi.org/10.1007/s40264-0180743-2

Reichling, J.J. and Kaplan, M.M., 1988. Clinical use of serum enzymes in liver disease. Dig. Dis. Sci., 33: 1601-1614. https://doi.org/10.1007/BF01535953

Rocco, A., Compare, D., Angrisani, D., Zamparelli, M.S. and Nardone, G., 2014. Alcoholic disease: Liver and beyond. World J. Gastroenterol., 20: 14652-14659. https://doi.org/10.3748/wjg.v20. i 40.14652

Selvaratnam, J. and Robaire, B., 2016. Effects of aging and oxidative stress on spermatozoa of superoxide-dismutase 1- and catalase-null mice. Biol. Reprod., 95: 60-60. https://doi.org/10.1095/ biolreprod.116.141671

Sid, B., Verrax, J. and Calderon, P.B., 2013. Role of oxidative stress in the pathogenesis of alcoholinduced liver disease. Free Radic. Res., 47: 894904. https://doi.org/10.3109/10715762.2013.8194 28

Sijben, J.W. and Calder, P.C., 2007. Differential immunomodulation with long-chain n-3 pufa in 
health and chronic disease. Proc. Nutr. Soc., 66: 237 259. https://doi.org/10.1017/S0029665107005472

Simopoulos, A.P., 2002. Omega-3 fatty acids in inflammation and autoimmune diseases. J. Am. Coll. Nutr., 21: 495-505. https://doi.org/10.1080/0 7315724.2002 .10719248

Song Z., Deaciuc, I., Song, M., Lee, D., Liu, Y. and McClain, X.J.C., 2010. Silymarin protects against acute ethanol-induced hepatotoxicity in mice. Alcohol. Clin. Exp. Res., 30: 407-413. https://doi. org/10.1111/j.1530-0277.2006.00063.x

Vega, M., Verma, M., Beswick, D., Bey, S., Hossack, J., Merriman, N., Shah, A. and Navarro, V., 2017. The incidence of drug- and herbal and dietary supplement-induced liver injury: Preliminary findings from gastroenterologist-based surveillance in the population of the state of delaware. Drug Saf., 40: 783-787. https://doi.org/10.1007/s40264017-0547-9

Vinson, J.A. and Cai, Y., 2012. Nuts, especially walnuts, have both antioxidant quantity and efficacy and exhibit significant potential health benefits. $F d$. Funct., 3: 134-140. https://doi.org/10.1039/ C2FO10152A

Wakimfleming, J. and Mullen, K.D., 2005. Longterm management of alcoholic liver disease. Clin. Liver Dis., 9: 135-149. https://doi.org/10.1016/j. cld.2004.10.001

Wang, M., Sun, J., Jiang, Z., Xie, W. and Zhang, X., 2015. Hepatoprotective effect of kaempferol against alcoholic liver injury in mice. Am. J. Chinese Med., 43: 241-254. https://doi.org/10.1142/ S0192415X15500160

Willis, L.M., Bielinski, D.F., Fisher, D.R., Matthan, N.R. and Joseph, J.A., 2010. Walnut extract inhibits lps-induced activation of bv-2 microglia via internalization of tlr4: Possible involvement of phospholipase d2. Inflammation, 33: 325-333. https://doi.org/10.1007/s10753-010-9189-0

Xu, G., Xiao, Y., Zhang, Q., Zhou, M. and Liao, S., 2018. Hepatoprotective natural triterpenoids. Eur. J. med. Chem., 145: 691-716. https://doi.org/10.1016/j. ejmech.2018.01.011

Zarate, R., Jabervazdekis, N.E., Tejera, N., Perez, J.A. and Rodriguez, C., 2017. Significance of long chain polyunsaturated fatty acids in human health. Clin. Transl. Med., 6: 25. https://doi.org/10.1186/ s40169-017-0153-6

Zhao, H., Li, J., Zhao, J., Chen, Y., Ren, C. and Chen, Y., 2018. Antioxidant effects of compound walnut oil capsule in mice aging model induced by d-galactose. Fd. Nutr. Res., 62: 1371. https://doi. org/10.29219/fnr.v62.1371 